\title{
Produtividade de forragem e morfogênese de Brachiaria brizantha cv. Marandu sob níveis de nitrogênio
} \author{
Seixas Santos 5 \\ ${ }^{I}$ Eng. Agr., D.Sc., Pesquisador da Embrapa Roraima, Boa Vista, RR, Brasil. \\ ${ }^{2}$ Zootecnista, D.Sc., Pesquisador da Embrapa Clima Temperado, Pelotas, RS, Brasil. \\ ${ }^{3}$ Zootecnista, D.Sc., Pesquisador da Embrapa Meio-Norte, Parnaíba, PI, Brasil. \\ ${ }^{4}$ Méd. Vet., Pesquisador da Embrapa Meio-Norte, Parnaíba, PI, Brasil. \\ ${ }^{5}$ Eng. Agr., D.Sc., Pesquisador da Embrapa Meio-Norte, Parnaíba, PI, Brasil. \\ *Autor para correspondência, E-mail: newtonlucena@yahoo.com.br \\ **in memorian
}

Newton de Lucena Costa ${ }^{1 *}$, Claudio Ramalho Townsend ${ }^{2 * *}$, Fabíola Helena dos Santos Fogaça $^{3}$, João Avelar Magalhães ${ }^{4}$, Amaury Burlamaqui Bendahan ${ }^{1}$, Francisco José de

\begin{abstract}
RESUMO. O efeito da adubação nitrogenada $\left(0,60,120,180\right.$ e $240 \mathrm{~kg}$ de $\mathrm{N}$ ha $\left.{ }^{-1}\right)$ sobre a produção de forragem e características morfogênicas e estruturais de Brachiaria brizantha cv. Marandu foi avaliado em condições de campo. A adubação nitrogenada afetou positiva e significativamente $(\mathrm{P}<0,05)$ a produção de matéria seca verde $(\mathrm{MSV}), \mathrm{o}$ perfilhamento, a senescência e as características morfogênicas e estruturais da gramínea. Os maiores rendimentos de MSV, taxa de expansão foliar, tamanho médio de folhas, número de folhas perfilho ${ }^{-1} \mathrm{e}$ índice de área foliar foram obtidas com a aplicação de 221,$5 ; 206,9 ; 188,6 ; 180,5$ e $205,5 \mathrm{~kg}^{\mathrm{de}} \mathrm{N} \mathrm{ha}^{-1}$, respectivamente. A eficiência de utilização de $\mathrm{N}$ foi inversamente proporcional às doses de $\mathrm{N}$ aplicadas.
\end{abstract}

Palavras chave: folhas, matéria seca, perfilhamento, senescência

\section{Forage yield and morphogenesis of Brachiaria brizantha cv. Marandu under nitrogen levels}

\begin{abstract}
The effect of nitrogen levels $\left(0,60,120,180\right.$ and $240 \mathrm{~kg}$ of $\left.\mathrm{N} \mathrm{ha}{ }^{-1}\right)$ on green dry matter (GDM) yield and morphogenetic and structural characteristics of Brachiaria brizantha cv. Marandu, was evaluated under field conditions. Nitrogen fertilization increased significantly $(\mathrm{P}<0.05)$ GDM yields, tillering, senescence and grass morphogenetic and structural characteristics. Maximum GDM yields, leaf elongation rates, leaf length, number of leaves plant ${ }^{-1}$ and leaf area index were obtained with the application of $221.5 ; 206.9 ; 188.6 ; 180.5$ and $205.5 \mathrm{~kg}$ of $\mathrm{N} \mathrm{ha}^{-1}$, respectively. The nitrogen efficiency utilization was inversely proportional to the increased nitrogen levels.
\end{abstract}

Keywords: dry matter, leaves, senescence, tillering

\section{Introdução}

Em Roraima, a pecuária é uma atividade econômica em plena expansão e as pastagens cultivadas constituem o principal recurso forrageiro para a alimentação dos rebanhos. O fogo é uma prática comumente utilizada no manejo das pastagens, pois apresenta baixo custo e fácil aplicação. Sua principal finalidade é a eliminação da biomassa seca acumulada e não consumida pelos animais durante o período de estiagem, proporcionando uma rebrota mais tenra, palatável e de melhor valor nutritivo, notadamente, em períodos de escassez de forragem (Costa et al., 2013). A queima incorpora, sob a forma de cinzas, todos os nutrientes não voláteis da biomassa, o que implica em aumento do $\mathrm{pH}$ e da fertilidade do solo, favorecendo $\mathrm{o}$ estabelecimento $\mathrm{e}$ crescimento das pastagens. No entanto, a alta 
fertilidade é temporária, pois o nitrogênio $(\mathrm{N})$ pode ser perdido por lixiviação, volatilização ou imobilização, sendo a sua deficiência apontada como uma das principais causas da degradação das pastagens (Costa et al., 2009).

As pastagens cultivadas, notadamente as formadas exclusivamente com gramíneas, necessitam de uma fonte para a reposição do $\mathrm{N}$ (química ou biológica), com o objetivo de manter a produção de forragem, e consequentemente evitar sua degradação (Nabinger \& Carvalho, 2009). O N é o principal nutriente para a manutenção da produtividade e persistência de uma pastagem de gramínea, sendo o principal constituinte das proteínas que participam ativamente na síntese dos compostos orgânicos que formam a estrutura do vegetal, sendo responsável por características estruturais da planta (tamanho de folha, densidade de perfilho e folhas por perfilho), além de características morfogênicas (taxas de aparecimento, alongamento e senescência foliar) (Lemaire et al., 2011, Santos et al., 2012). Nos solos deficientes em $\mathrm{N}$, o crescimento e o desenvolvimento da planta tornam-se lentos, a produção de perfilhos é negativamente afetada e o teor de proteína tornase deficiente para o atendimento das exigências do animal (Costa et al., 2009).

Neste trabalho foram avaliados os efeitos da adubação nitrogenada sobre a produção de forragem e características morfogênicas e estruturais de Brachiaria brizantha cv. Marandu, nos cerrados de Roraima.

\section{Material e Métodos}

$\mathrm{O}$ ensaio foi conduzido no Campo Experimental da Embrapa Roraima, localizado em Boa Vista, durante o período de maio a setembro de 2013. O solo da área experimental é um Latossolo Amarelo, textura média, com as seguintes características químicas, na profundidade de $0-20 \mathrm{~cm}: \mathrm{pH}_{\mathrm{H} 2 \mathrm{O}}=4,7 ; \mathrm{P}=1,8$ $\mathrm{mg} / \mathrm{kg} ; \mathrm{Ca}+\mathrm{Mg}=0,98 \mathrm{cmol}_{\mathrm{c}} \cdot \mathrm{dm}^{-3} ; \mathrm{K}=0,03$ $\mathrm{cmol}_{\mathrm{c}} \cdot \mathrm{dm}^{-3} ; \mathrm{Al}=0,58 \mathrm{cmol}_{\mathrm{c}} \mathrm{dm}^{-3} ; \mathrm{H}+\mathrm{Al}=2,64$ $\mathrm{cmol}_{\mathrm{c}} \cdot \mathrm{dm}^{-3}$.

$\mathrm{O}$ delineamento experimental foi inteiramente ao acaso com três repetições. Os tratamentos consistiram de cinco níveis de nitrogênio $(0 ; 60$; $120 ; 160$ e $240 \mathrm{~kg}$ de $\mathrm{N} / \mathrm{ha}$ ), aplicados sob a forma de ureia. $\mathrm{O}$ tamanho das parcelas foi de 2,0 x 2,0 m, sendo a área útil de $1,0 \mathrm{~m}^{2}$. A aplicação do nitrogênio foi parcelada em duas vezes, sendo metade quando da roçagem da pastagem, ao início do experimento, e metade decorridos 45 dias. Durante o período experimental foram realizados três cortes a intervalos de 45 dias.

Os parâmetros avaliados foram rendimento de matéria seca verde (MSV), eficiência de utilização de nitrogênio (EUN), número de perfilhos $\mathrm{m}^{-2}$ (NP), número de folhas perfilho ${ }^{-1}$ (NFP), taxa de aparecimento de folhas (TAF), taxa de expansão foliar (TEF), taxa de senescência foliar (TSF), tamanho médio de folhas (TMF) e índice de área foliar (IAF). A EUN foi determinada relacionando-se $\mathrm{O}$ rendimento de MSV com a dose aplicada de N. A TEF e a TAF foram calculadas dividindo-se o comprimento acumulado de folhas e o número total de folhas no perfilho, respectivamente, pelo período de rebrota. O TMF foi determinado pela divisão do alongamento foliar total do perfilho pelo número de folhas. Para o cálculo da área foliar foram coletadas amostras de folhas verdes completamente expandidas, procurando-se obter uma área entre 200 e $300 \mathrm{~cm}^{2}$. As amostras foram digitalizadas e a área foliar estimada com o auxílio de planímetro ótico eletrônico (Li-Cor 3100C). Posteriormente, as amostras foram levadas à estufa com ar forçado a $65^{\circ} \mathrm{C}$ até atingirem peso constante, obtendo-se a MS foliar. A área foliar específica (AFE) foi determinada através da relação entre a área de folhas verdes e a sua MS $\left(\mathrm{m}^{2} / \mathrm{g}\right.$ MS foliar). O IAF foi determinado a partir do produto entre a MS total das folhas verdes $\left(\mathrm{g}\right.$ de $\left.\mathrm{MS} / \mathrm{m}^{2}\right)$ pela AFE $\left(\mathrm{m}^{2} / \mathrm{g}\right.$ de MS foliar). A TSF foi obtida dividindo-se o comprimento da folha que se apresentava de coloração amarelada ou necrosada pela idade de rebrota.

Os dados foram submetidos à análise de variância e de regressão considerando o nível de significância de 5\% de probabilidade. Para se estimar a resposta dos parâmetros avaliados aos níveis de $\mathrm{N}$, a escolha dos modelos de regressão baseou-se na significância dos coeficientes linear e quadrático, por meio do teste " $t$ ", de Student, ao nível de $5 \%$ de probabilidade.

\section{Resultados e Discussão}

A adubação nitrogenada afetou $(\mathrm{P}<0,05)$ os rendimentos de MSV, sendo a relação quadrática e o máximo valor estimado com a aplicação de $221,5 \mathrm{~kg}$ de $\mathrm{N} \mathrm{ha}^{-1}$ (3.772 $\mathrm{kg}$ de MSV ha $\left.{ }^{-1}\right)$. A eficiência de utilização de $\mathrm{N}$ foi inversamente proporcional às doses de $\mathrm{N}$ aplicadas, sendo a relação linear e definida pela equação $Y=52,13$ - 
$0,1641 X\left(r^{2}=0,89\right)$. (Tabela 1). Tendências semelhantes foram reportadas por Costa et al. (2009) que constataram máximos rendimentos de forragem de Brachiaria brizantha cv. Xaraés e $B$. decumbens com a aplicação de 175,2 e $232,9 \mathrm{~kg}$ de $\mathrm{N} \mathrm{ha}^{-1}$, respectivamente, contudo as maiores taxas de eficiência de utilização do $\mathrm{N}$ foram registradas sob doses entre 80 e $120 \mathrm{~kg}$ de $\mathrm{N} \mathrm{ha}^{-1}$. A EUN é afetada pelas espécies forrageiras, estádio de desenvolvimento das plantas, doses aplicadas e seu fracionamento, frequência de utilização das pastagens, fatores ambientais e fertilidade do solo. Reduções na EUN e RAN podem estar associadas a perdas de $\mathrm{N}$ por lixiviação, volatilização de $\mathrm{NH}_{3}$ e desnitrificação, notadamente com a utilização de elevadas doses de $\mathrm{N}$ e sob condições de alta umidade do solo (Nabinger \& Carvalho, 2009). O NP foi positiva e linearmente afetado pela adubação nitrogenada
$\left(\mathrm{Y}=694+1,1867 \mathrm{X}-\mathrm{r}^{2}=0,94\right)$. A correlação entre NP e rendimento de MS foi positiva e significativa $(\mathrm{r}=0,95 ; \mathrm{P}<0,01)$, a qual explicou em $90 \%$ os incrementos verificados nos rendimentos de MS da gramínea, em função da adubação nitrogenada (Tabela 1).

$\mathrm{O} \mathrm{N}$ interfere intensamente na ativação dos tecidos meristemáticos (gemas axilares), pois seu déficit aumenta o número de gemas dormentes, enquanto que seu adequado suprimento permite o máximo perfilhamento da gramínea (Nabinger \& Carvalho, 2009). Os perfilhos individuais têm duração de vida limitada e variável, em função de fatores bióticos e abióticos, e sua população pode ser mantida por uma contínua reposição dos perfilhos mortos, sendo este comportamento o ponto-chave para a perenidade das gramíneas (Lemaire et al., 2011, Santos et al., 2012)

Tabela 1. Rendimento de matéria seca verde (MSV - $\mathrm{kg} \mathrm{ha}^{-1}$ ), eficiência de utilização do nitrogênio (EUN - kg de $\mathrm{MSV} / \mathrm{kg}$ de $\mathrm{N} \mathrm{ha}^{-1}$ ), número de perfilhos $\mathrm{m}^{-2}$ (NP), número de folhas perfilho-1 (NFP), tamanho médio de folhas (TMF - cm), índice de área foliar (IAF), taxa de aparecimento de folhas (TAF - folhas perfilho-1 dia $^{-1}$ ), taxa de expansão foliar (TEF - cm perfilho ${ }^{-1}$ dia $^{-1}$ ) e taxa de senescência foliar (TSF - cm perfilho-1 dia $^{-1}$ ) de Brachiaria brizantha cv. Marandu, em função da adubação nitrogenada. Médias de três cortes.

\begin{tabular}{|c|c|c|c|c|c|c|}
\hline \multirow[t]{2}{*}{ Variáveis } & \multicolumn{5}{|c|}{ Doses de $\mathrm{N} \mathrm{ha}^{-1}$} & \multirow{2}{*}{ Equação de Regressão } \\
\hline & 0 & 60 & 120 & 180 & 240 & \\
\hline MSV & 1.817 & 2.789 & 3.211 & 3.857 & 3.711 & $Y=1.818+17,731 X-0,0402 X^{2}\left(R^{2}=0,97\right)$ \\
\hline EUN & --- & 46,5 & 26,7 & 21,4 & 15,5 & $Y=52,13-0,1641 X\left(r^{2}=0,89\right)$ \\
\hline NP & 672 & 773 & 859 & 935 & 947 & $Y=694+1,1867 X\left(r^{2}=0,94\right)$ \\
\hline NFP & 3,98 & 4,57 & 5,11 & 5,39 & 5,08 & $Y=3,92+0,01484 X-0,000041 X^{2}\left(R^{2}=0,97\right)$ \\
\hline TMF & 19,6 & 22,5 & 24,8 & 26,7 & 25,1 & $Y=19,4+0,06752 X-0,000179 X^{2}\left(R^{2}=0,98\right)$ \\
\hline IAF & 2,17 & 2,99 & 3,55 & 3,99 & 3,81 & $Y=2,14+0,01726 X-0,000042 X^{2}\left(R^{2}=0,98\right)$ \\
\hline TAF & 0,0884 & 0,1016 & 0,1136 & 0,1198 & 0,1129 & $\left.\mathrm{Y}=0,09382+0,00011185 \mathrm{X}-\mathrm{r}^{2}=0,87\right)$ \\
\hline TEF & 1,73 & 2,29 & 2,82 & 3,15 & 2,83 & $Y=1,61+0,01491 X-0,000036 X^{2}\left(R^{2}=0,98\right)$ \\
\hline TSF & 0,098 & 0,131 & 0,157 & 0,175 & 0,198 & $Y=0,1046+0,00047 X\left(r^{2}=0,98\right)$ \\
\hline
\end{tabular}

A relação entre adubação nitrogenada e o NFP foi ajustada ao modelo quadrático de regressão e o máximo valor obtido com a aplicação de 180,5 $\mathrm{kg}$ de $\mathrm{N} \mathrm{ha}^{-1}\left(5,25\right.$ folhas perfilho $\left.{ }^{-1}\right)$. Os valores obtidos foram superiores aos reportados por Luna et al. (2012) para B. brizantha cvs. Xaraés e Piatã, que estimaram 3,91 e 4,33 folhas verdes perfilho $^{-1}$, respectivamente. O principal efeito do $\mathrm{N}$ sobre o NFP seria o aumento na duração de vida das folhas por meio da manutenção de maior capacidade fotossintética por períodos mais longos, sem que haja remobilização interna significativa do $\mathrm{N}$ das folhas mais velhas (Garcez
Neto et al., 2002, Nabinger \& Carvalho, 2009). Em Panicum maximum cv. Massai, a eficiência fotossintética foi diretamente proporcional aos níveis de $\mathrm{N}\left(0,150,300,450\right.$ e $600 \mathrm{mg}$ de $\mathrm{N} \mathrm{dm}^{-3}$ de solo), como consequência da maior relação fotossíntese/transpiração, redução da condutância estomática e aumento do índice relativo de clorofila. Como o NFP é uma característica genética da espécie, pode-se inferir que plantas recebendo $\mathrm{N}$ irão atingir seu número máximo de folhas mais precocemente, em relação às nãoadubadas, permitindo a possibilidade de colheitas mais frequentes, a fim de evitar perdas por 
senescência foliar (Alexandrino et al., 2011).

A TAF foi positiva e linearmente afetada pela adubação nitrogenada $(\mathrm{Y}=0,09382+$ $\left.0,00011185 X-r^{2}=0,87\right)$, enquanto que para a TEF, o TMF e o IAF o efeito foi quadrático e os valores máximos obtidos com a aplicação de 206,9; 188,6 e 205,5 kg de $\mathrm{N} \mathrm{ha}^{-1}$. A correlação entre rendimento de MS e a TEF foi positiva e significativa $(r=0,93 ; \mathrm{P}<0,01)$, enquanto que com a TAF a correlação foi positiva, porém não significativa $(\mathrm{r}=0,75 ; \mathrm{P}>0,05)$. Alexandrino et al. (2011) constataram efeito quadrático da adubação nitrogenada $(0,45,90,180$ e $360 \mathrm{mg}$ de $\mathrm{N} \mathrm{kg} \mathrm{solo}{ }^{-1}$ ) sobre a TAF, TEF e TMF de $B$. brizantha cv. Marandu, estimando os valores máximos com a aplicação de 335; 325 e $347 \mathrm{mg}$ $\mathrm{N} / \mathrm{kg}$ solo, respectivamente. Para Lemaire et al. (2011), a TEF, ao responder ao suprimento de N, seria o principal agente modificador da TAF. Folhas sucessivas aparecendo em níveis de inserção muito próximos, mas sob elevadas taxas de alongamento, suportadas pelo suprimento adicional de $\mathrm{N}$, estabeleceriam maior TAF em decorrência de seu efeito positivo sobre o número e o tamanho das células produzidas na zona de divisão celular. A TEF e a TAF apresentam correlação negativa, indicando que quanto maior a TAF, menor será o tempo disponível para o alongamento das folhas (Garcez Neto et al., 2002, Santos et al., 2012). Neste trabalho a correlação entre estas duas variáveis foi positiva e significativa $(\mathrm{r}=0,91 ; \mathrm{P}<0,05)$, possivelmente, como consequência das condições ambientais favoráveis, as quais permitiram que as plantas expressassem seu máximo potencial de crescimento.

A TSF foi diretamente proporcional às doses de $\mathrm{N}$ aplicadas, sendo a relação ajustada ao modelo linear de regressão $(\mathrm{Y}=0,1046+$ $\left.0,00047 \mathrm{X}-\mathrm{r}^{2}=0,98\right)$. Resultados semelhantes foram reportados por Costa et al. (2009) para $B$. brizantha cv. Marandu, que constataram maiores TSF com a aplicação de $120\left(0,159 \mathrm{~cm}_{\text {perfilho }}{ }^{-1}\right.$ dia $\left.^{-1}\right), 160\left(0,168 \mathrm{~cm}_{\text {perfilho }}{ }^{-1} \mathrm{dia}^{-1}\right)$ e $180 \mathrm{~kg}$ de $\mathrm{N}$ ha $^{-1}$ ano $^{-1}\left(0,175 \mathrm{~cm}\right.$ perfilho $\left.{ }^{-1} \mathrm{dia}^{-1}\right)$. A senescência é um processo natural que caracteriza a última fase de desenvolvimento de uma folha, o qual é iniciado após a completa expansão das primeiras folhas, cuja intensidade se acentua progressivamente com o aumento da área foliar, a qual implica no sombreamento das folhas inseridas na porção inferior do colmo (Silva et al., 2009, Lemaire et al., 2011). A senescência apesar do efeito negativo sobre a qualidade da forragem representa um importante processo fisiológico no fluxo de tecidos da gramínea, pois em torno de 35; 68; 86 e $42 \%$ do nitrogênio, fósforo, potássio e magnésio, respectivamente, podem ser reciclados das folhas senescentes e utilizados para a produção de novos tecidos foliares (Sarmiento et al., 2006, Costa et al., 2013).

\section{Conclusões}

A adubação nitrogenada afeta positivamente o rendimento de forragem e as características morfogênicas e estruturais da gramínea. A eficiência de utilização de nitrogênio é inversamente proporcional às doses aplicadas. $\mathrm{O}$ processo de senescência foliar da gramínea é acelerado com o aumento das doses de nitrogênio.

\section{Referências Bibliográficas}

Alexandrino, E., Cândido, M. J. D. \& Gomide, J. A. (2011). Fluxo de biomassa e taxa de acúmulo de forragem em capim| Mombaça mantido sob diferentes alturas. Revista Brasileira de Saúde e Produção Animal, 12, 59-71.

Costa, N. L., Gianluppi, V., Braga, R. M. \& Bendahan, A. B. (2009). Alternativas tecnológicas para a pecuária de Roraima. Boa Vista: Embrapa Roraima, 35p. (Documentos 19).

Costa, N. L., Moraes, A., Monteiro, A. L. G., Motta, A. C. V., Oliveira, R. A. \& Rodrigues, A. N. A. (2013). Forage productivity and morphogenesis of Axonopus aureus under different nitrogen fertilization rates. Revista Brasileira de Zootecnia, 42, 541-548.

Garcez Neto, A., Nascimento Júnior, D. \& Regazzi, A. J. (2002). Respostas morfogênicas $\mathrm{e}$ estruturais de Panicum maximum cv. Mombaça sob diferentes níveis de adubação nitrogenada e alturas de corte. Revista Brasileira de Zootecnia, 31, 1890-1900.

Lemaire, G., Hodgson, J. \& Chabbi, A. (2011). Grassland productivity and ecosystem services. Cabi, Wallingford. 312p.

Luna, A. A., Difante, G. S., Araújo, I. M. M. \& Lima, C. L. D. (2012). Características morfogênicas de gramíneas forrageiras no Nordeste do Brasil. Revista Científica de Produção Animal, 14, 138-141. 
Nabinger, C. \& Carvalho, P. C. F. (2009). Ecofisiología de sistemas pastoriles: aplicaciones para su sustentabilidad. Agrociencia, 13, 18-27.

Santos, M. R., Fonseca, D. M., Gomes, V. M., Silva, S. P., Silva, G. P. \& Reis, M. (2012). Correlações entre características morfogênicas e estruturais em pastos de capim-braquiária. Ciência Animal Brasileira, 13, 49-56.

Sarmiento, G., Silva, M. P., Naranjo, M. E. \& Pinillos, M. (2006). Nitrogen and phosphorus as limiting factors for growth and primary production in a flooded savanna in the Venezuelan Llanos. Journal of Tropical Ecology, 22, 203-212.

Silva, C. C. F., Bonomo, P., Pires, A. J. V.,
Maranhão, C. M. A., Patês, N. M. S. \& Santos, L. C. (2009). Características morfogênicas e estruturais de duas espécies de braquiária adubadas com diferentes doses de nitrogênio. Revista Brasileira de Zootecnia, $38,657-661$.

\section{Article History:}

Received 15 July 2016

Accepted 8 August 2016

Available on line 24 August 2016

License information: This is an open-access article distributed under the terms of the Creative Commons Attribution License, which permits unrestricted use, distribution, and reproduction in any medium, provided the original work is properly cited. 